\title{
Malignant Psoas Syndrome Demonstrated by PET/CT in the Context of Metastatic Non Small Cell Lung Cancer
}

\author{
Michael Jerome McKay ${ }^{1, ~ *, ~ A a r o n ~ C h i n d e w e r e ~}{ }^{1}$, Laura Wise ${ }^{2}$, Fraser Brown ${ }^{3}$, \\ Kim Louise Taubman ${ }^{4}$, Timothy Michael McKay ${ }^{5}$ \\ ${ }^{1}$ Departments of Radiation and Medical Oncology, North West Cancer Centre, Burnie, Australia \\ ${ }^{2}$ Northern Tasmanian Pathology Service, Launceston General Hospital, Launceston, Australia \\ ${ }^{3}$ I-Med Medical Imaging, Launceston General Hospital, Launceston, Australia \\ ${ }^{4}$ Department of Medical Imaging, St Vincents Hospital, Melbourne, Australia \\ ${ }^{5}$ Department of Precision Medicine, Monash University, Melbourne, Australia
}

Email address:

michael.mckay@ths.tas.gov.au (M. J. McKay)

${ }^{*}$ Corresponding author

\section{To cite this article:}

Michael Jerome McKay, Aaron Chindewere, Laura Wise, Fraser Brown, Kim Louise Taubman, Timothy Michael McKay. Malignant Psoas Syndrome Demonstrated by PET/CT in the Context of Metastatic Non Small Cell Lung Cancer. International Journal of Clinical Oncology and Cancer Research. Vol. 6, No. 3, 2021, pp. 141-144. doi: 10.11648/j.jicocr.20210603.17

Received: July 26, 2021; Accepted: August 4, 2021; Published: August 18, 2021

\begin{abstract}
The significant cancer pain syndrome of malignant psoas syndrome (MPS), was first reported as a neurooncologic occurrence in 1990. The Syndrome is characterised by malignant infiltration of a psoas muscle, either directly or by involvement with haematogenously disseminated metastatic cancer. Direct involvement has been from primary tumours of the psoas muscle, or by extension from malignant paraaortic lymphadenopathy. Treatment is difficult, complex analgesia is essential, and benefits have been achieved from chemotherapy, radiotherapy and in select cases, surgery. Here, the case of a 60 year old female who presented with three months of moderate dyspnoea, low back and left inguinal region pain/numbness and lassitude, is reported. Chest radiograph and CT showed a large anterior mediastinal mass. FDG PET/CT revealed the mass to be intensely avid, with heterogeneous central areas of photopaenia. Avid lymphadenopathy was also present in two mediastinal nodal stations. Subdiaphragmatically, there was a metabolically FDG avid soft tissue mass in the superior part of the left psoas muscle, presumably accounting for her pain. There was no FDG avidity elsewhere. Core biopsy of the psoas mass revealed adenocarcinoma of probable lung origin. The patient responded symptomatically to intermediate dose radiation therapy. The pathophysiology of MPS is discussed and the range of cancer types associated with the Syndrome is updated.
\end{abstract}

Keywords: Malignant Psoas Syndrome, MPS, PET/CT, Non Small-cell Lung Cancer

\section{Introduction}

Malignant Psoas Syndrome (MPS) was first reported as a neurooncologic occurrence in 1990 [1]. The Syndrome is characterised by malignant infiltration of a psoas muscle and adjacent lumbar nerves, either directly or by involvement with haematogenously disseminated metastatic cancer. Direct involvement has been from rare primary tumours of the psoas muscle, or more commonly by extension from malignant paraaortic lymphadenopathy. Patients usually have severe, refractory pain and characteristically demonstrate a flexion deformity of the ipsilateral hip and features of proximal lumbosacral plexopathy. MPS is a devastating pain syndrome with a poor outlook, although responses to both radiotherapy $[2,3]$ and chemotherapy $[4,5]$ are reported.

Here, the case of a woman with metastatic non small cell lung cancer who manifested MPS is presented, the aetiology of MPS is discussed and the range of cancer types associated with the Syndrome is updated. 


\section{Main Body}

A 60 year old female smoker presented with three months of moderate dyspnoea, low back and left inguinal region pain/numbness and lassitude. The pain at both sites was exacerbated by walking and weight-bearing. She also had skin tingling in the left upper anterior thigh, which was hyperaesthetic, and showed reduced pinprick sensation. Otherwise, neurological examination, including the left leg, was normal, as was general physical examination. In particular, the left psoas muscle stretch test was negative.

Chest radiograph demonstrated volume loss in the left hemithorax, with an elevated left hemidiaphragm, peribronchial thickening and patchy airspace infiltrates in the left lower zone. CT showed a large anterior mediastinal mass measuring $55 \mathrm{~mm}$ in maximum diameter, anterior to the aortic arch and pulmonary trunk. Laterally, the mass abutted and potentially invaded the mediastinal pleura, with extension into the anterior segment of the left upper lobe. FDG PET/CT revealed the mass to be intensely avid (SUV max 21.4), with heterogeneous central areas of photopaenia, presumably representing central necrosis. Avid lymphadenopathy was also present lateral to the aortic arch, as well as posterior to the brachiocephalic vein. Subdiaphragmatically, there was a metabolically avid soft tissue mass measuring $5 \mathrm{~cm}$ in maximal diameter, in the superior part of the left psoas muscle (Figure 1). There was no FDG avidity above background elsewhere. The patient's symptoms, her physical signs and imaging investigations were consistent with left psoas muscle and lumbar plexus invasion/involvement by metastatic lung cancer. Core biopsy of the PET/CT-avid mass in the upper left psoas muscle showed infiltration by epithelioid cells with moderate to abundant cytoplasm, with focal gland-like structures. Immunohistochemically, the tumour cells were TTF1-positive and PAX8-negative, in keeping with metastatic adenocarcinoma of primary lung origin (Figure 2).
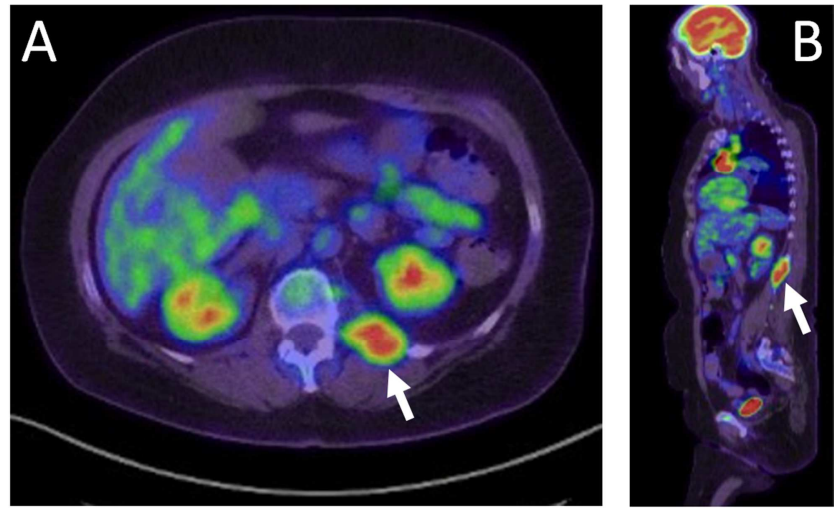

McKay et al. Malignant Psoas Syn.

Figure 1. A. Axial PET/CT slice at the level of the first lumbar vertebra. There is intense FDG uptake in the upper part of the left psoas muscle (arrow), without vertebral destruction seen on the low-dose CT component (not shown). Physiological FDG uptake is seen in liver, kidneys, pancreatic tail and small intestine. B. Sagittal PET/CT slice showing the FDG-avid left psoas mass at the upper end of the muscle (arrow).
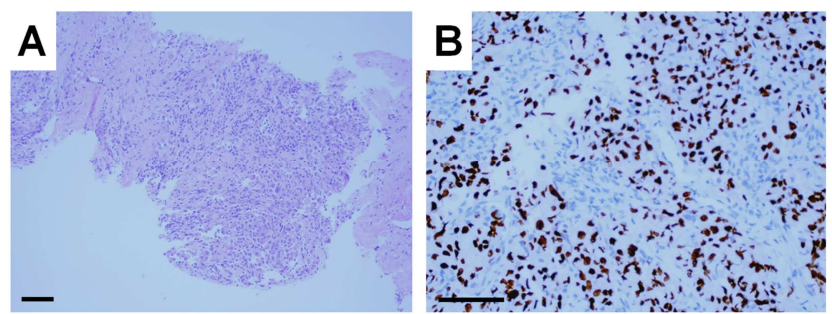

McKay et al. Malignant Psoas Syn.

Figure 2. A. Haematoxylin and eosin stain of left psoas muscle core biopsy, showing fibrous tissue infiltrated by epithelioid tumour cells with abundant cytoplasm and eccentric nuclei containing prominent nucleoli. Magnification x10. Bar: 100uM. B. TTF1 immunohistochemistry of the psoas biopsy, with positive staining in the nuclei of the tumour cells. Magnification x20. Bar: 100uM.
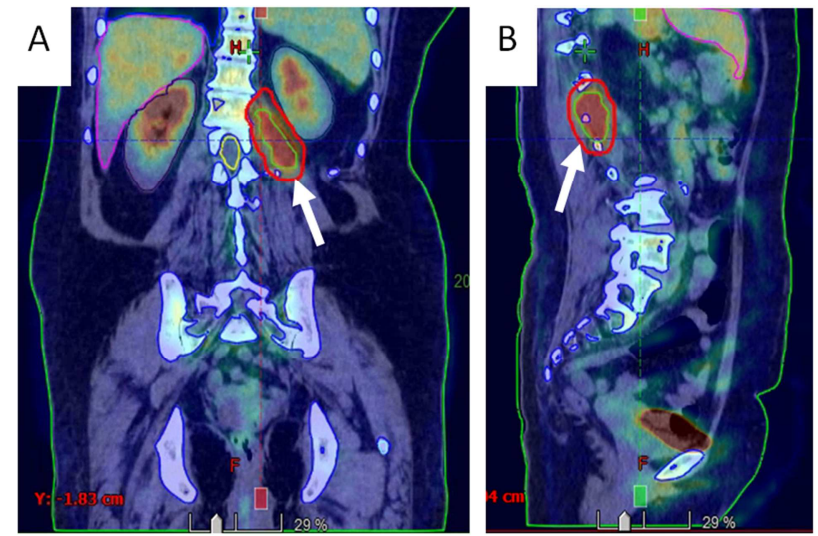

McKay et al. Malignant Psoas Syn.

Figure 3. A. Coronal radiotherapy planning CT-FDG PET fusion, showing FDG signal (inner orange signal), in left psoas muscle (white arrow). Green line: clinical target volume (CTV); red line: planning target volume (PTV). B. Sagittal view, same parameters.
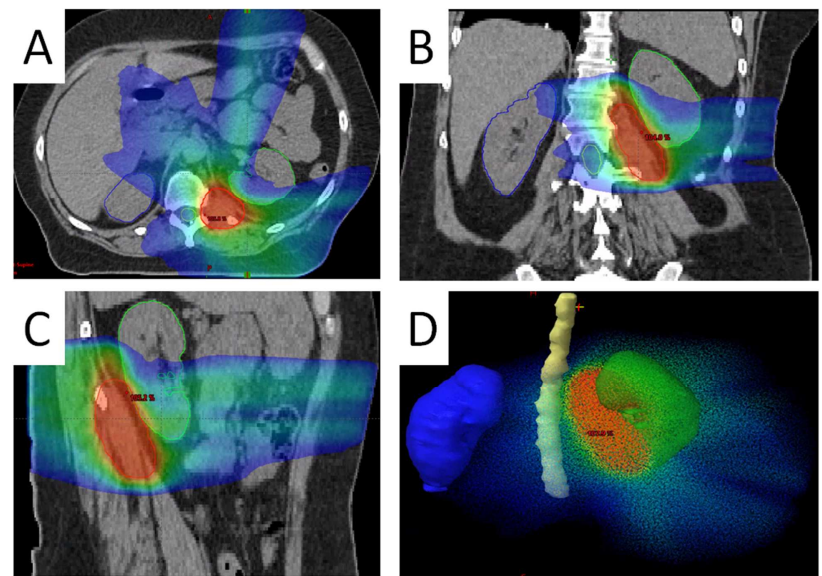

McKay et al. Malignant Psoas Syn.

Figure 4. Volumetric Modulated Arc Therapy (VMAT) technique used for the radiotherapeutic treatment of our MPS case. A. Axial CT slice showing radiotherapy target (red oval) and radiation dose wash across the target. Cool colours are lower doses (blue lowest; green intermediate), warm colours are higher doses. The target received $45 G y$ in 15 fractions over three weeks. $B$. Coronal CT slice showing proximity of the target to the left kidney. C. Sagittal $C T$ slice showing target in the superior portion of the psoas muscle. D. $3 D$ renders with dose cloud showing the relationship of the target (orange-red) to the kidneys and spinal cord. The left kidney received the higher dose, but still within organ tolerance. 
The patient was treated with a combination of systemic chemoimmunotherapy (with carboplatin, pemetrexed and pembroluzimab), as well as localised external beam radiotherapy to the left psoas lesion using VMAT (Volumetric Modulated Arc Therapy) (Figures 3, 4). The psoas region was treated with 45Gy in 15 fractions, a relatively high dose for non-SABR radiotherapy, since she had a good performance status, was relatively young, had solitary extrathoracic disease and the lesion was in a site where normal tissues would tolerate it. The patient responded symptomatically to intermediate dose radiation therapy (assessed at 3 months).

\section{Discussion}

MPS is in general an intractable and complex pain syndrome, affecting predominantly patients with metastatic epithelial cancers. The main clinical features are characteristic: lumbar/proximal leg pain with nociceptive and neuropathic and muscle spasm elements, flexion deformity of the ipsilateral hip and a positive psoas stretch test [1, 4]. Radiological/nuclear medicine examination with CT, MRI or more recently FDG-PET/CT [5], show, in the psoas muscle, characteristic density changes, expansion of all or part of the muscle and/or increase in FDG avidity. Changes may be bilateral. Such cases are typically associated with malignant paraaortic lymphadenopathy, and extension into the epidural space with resultant epidural neurological compression is a high risk (see below). Radiological/nuclear medicine investigations may obviously also show other sites of primary, regional or metastatic disease, most notably, enlarged paraaortic lymph nodes.

Since most reports of MPS are single case studies, its true incidence is difficult to determine. To date, 22 cases of MPS have been cumulatively reported) $[2,4]$ (Table 1).

In post-mortem studies, metastases to the psoas muscle are reported in 0.8 to $16 \%$ of cases $[12,13]$, although many of these were presumably subclinical and the psoas muscle may not have been specifically examined in routine autopsies in patients with metastatic cancer, leading to under-reporting bias.

The psoas muscle arises from the lateral parts of the $12^{\text {th }}$ thoracic vertebral body through to the $5^{\text {th }}$ lumbar vertebra. Its insertion is into the lesser trochanter of the femur and it is joined by the iliacus muscle in the pelvis. The lumbosacral nerve plexus interdigitates with the psoas muscle [14]. The role of psoas is as a thigh flexor. Invasion/irritation of the muscle hence causes hip flexion, sometimes fixed [4].

A secondary potential consequence of MPS is extension of tumour from psoas into intervertebral foraminae. Depending on the level of the lesion, this can lead to epidural spinal cord, conus medullaris or cauda equina compression. This is a well-recognised and dire consequence of MPS, occurring in up to $50 \%$ of cases with malignant involvement of the psoas muscle [15]. Our case did not show intervertebral tumour extension on $\mathrm{CT}$; further evaluation with the more sensitive MRI could have been considered.

Our case was the second case reported as a result of involvement of the psoas muscle by metastatic bronchial carcinoma [8]. This may seem surprising, as stage IV lung cancer is common, and it not uncommonly disseminates to pleiotropic locations. However, many MPS cases arise from invasion of the psoas muscle by malignant paraaortic lymphadenopathy, perhaps numerically overshadowing haematogenous spread to psoas. As mentioned above, epithelial malignancies account for the vast majority of cases of MPS [2, 4].

Table 1. Reported cases of MPS since 2017, when previously tabulated by McKay et al (2017). MPS cases prior to that were tabulated by Stevens et al (2010).

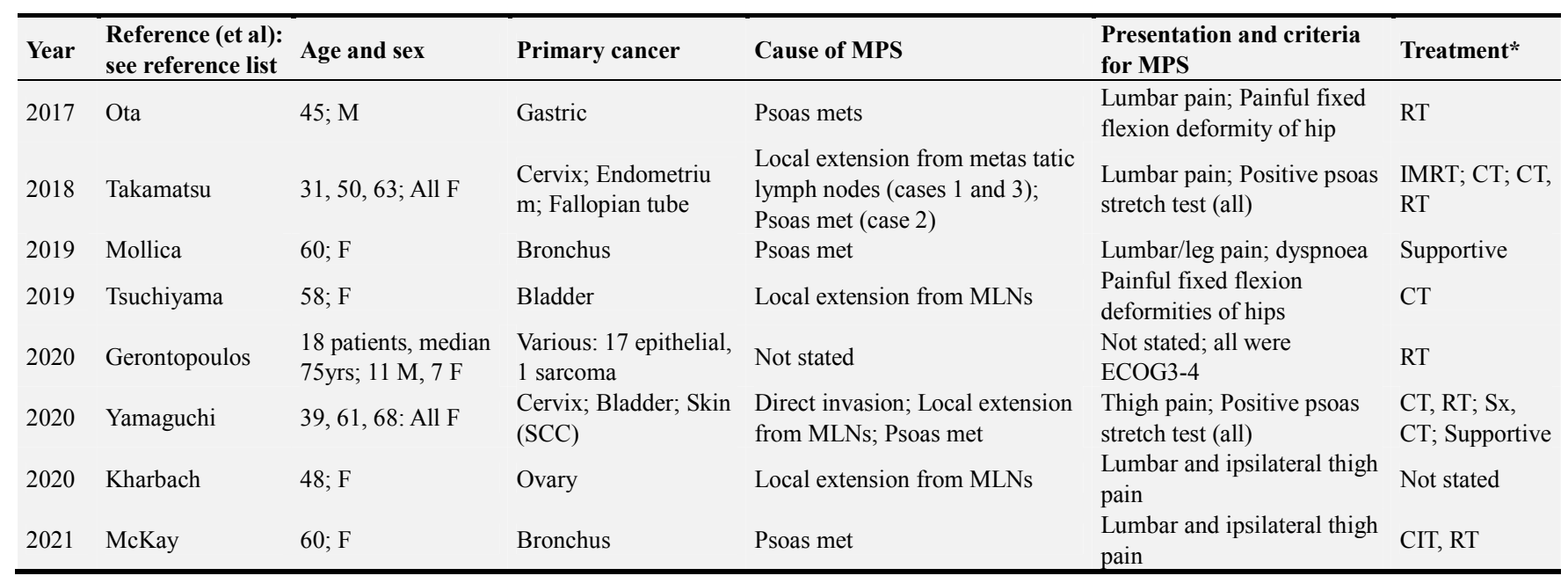

*All had at least opioid analgesia. Abbreviations: IMRT: intensity modulated radiotherapy; CT: chemotherapy; RT: radiotherapy; CIT: chemoimmunotherapy; met: metastases; ECOG: Eastern Cooperative Oncology Group; MLNs: metastatic lymph nodes; Sx: surgery; SCC: squamous cell carcinoma.

Although appropriate symptoms and signs were present in our case to support the diagnosis of MPS, she did not manifest a positive psoas stretch test. Presumably there was insufficient pathology within the muscle or lumbar plexus at the time of diagnosis to lead to psoas spasm.

An option apart from conventional radiotherapy to treat the psoas muscle metastasis in our case would have been SABR (stereotactic ablative body radiotherapy). Her disease would 
seem a good candidate for $\mathrm{SABR}$, being in a site easily verifiable with $\mathrm{CT}$, being of a suitable size and in a relatively radioresistant location. However, despite having an apparent extrathoracic oligometastasis, she unfortunately had, apart from the primary tumour, two sites of discrete nodal metastasis in the thorax. This overall clinical scenario is not considered oligometastatic disease [16] suitable for SABR.

MPS management is both general and cancer-specific. Non-oncological drug treatment is a cornerstone of therapy, as exemplified by the treatment of the four cases reported by Agar et al (2004) [17] (refer to their Table 1): analgesics (eg, acetaminophen, oxycodone, fentanyl, methadone, morphine), co-analgesics (eg, celebrex, naproxen), dexamethasone, sedatives (eg, diazepam), anticonvulsants (eg, sodium valproate, gabapentin, pregabalin). Analgesia delivered through epidural catheters can also have utility in MPS. One report described a patient with intractable bilateral flexion deformities in the hips, which was mitigating against her adequate positioning for radiotherapy [6]. In this case, epidural analgesia enabled enough degree of hip extension to facilitate her positioning for radiotherapy treatment. Other studies have also advocated epidural analgesia as an effective symptomatic measure to be considered in MPS [10].

For MPS, cancer-specific treatments of chemotherapy and radiotherapy have both been used with good effect (Table 1) $[3,4,7,17]$ and surgery has been used in some cases of localised disease uncomplicated by systemic disease, either alone or in combination with other modalities [2, 17].

\section{Conclusion}

MPS is typically an intractable pain syndrome in cases of advanced cancer, and fortunately is of relatively low incidence. The majority of cases are secondary to either haematogenous spread or spread from involved paraaortic lymph nodes. Its clinical features are characteristic and in general easily recognizable, including flexion deformities of the hip and positive psoas stretch test. An early multidisciplinary management approach is recommended, to yield the most satisfactory outcomes. In the case we present here there was a favourable early response to moderate dose local radiotherapy.

\section{Conflicts of Interest}

All the authors do not have any possible conflicts of interest.

\section{References}

[1] Stevens MJ and Gonet YM. Malignant psoas syndrome: recognition of an oncologic entity. Australas Radiol. 1990; 34: 150-154.
[2] McKay TA, Bishop S, McKay MJ. Primary psoas sarcoma causing malignant psoas syndrome: favourable response to radiotherapy. Ann Transl Med. 2017; 5: 105-108.

[3] Gerontopoulos A, Mosa E, Alongi F et al. Impact of Radiation Therapy on Pain Relief of Cancer Patients Affected by Malignant Psoas Syndrome: 26 Years of Experience. Indian J Palliat Care. 2020; 26: 348-351.

[4] Stevens MJ, Atkinson C, Broadbent AM. The malignant psoas syndrome revisited: case report, mechanisms and current therapeutic options. J Palliat Med. 2010; 13: 211-216.

[5] Basu S, Mahajan A. Psoas muscle metastasis from cervical carcinoma: Correlation and comparison of diagnostic features on FDG-PET/CT and diffusion-weighted MRI. World J Radiol. 2014; 6: 125-129.

[6] Ota T, Makihara M, Tsukuda $\mathrm{H}$ et al. Pain Management of Malignant Psoas Syndrome Under Epidural Analgesia During Palliative Radiotherapy. J Pain Palliat Care Pharmacother. 2017; 31: 154-157.

[7] Takamatsu S, Murakami K, Takaya H. Malignant psoas syndrome associated with gynecological malignancy: Three case reports and a review of the literature. Mol Clin Oncol. 2018 Jul; 9 (1): 82-86.

[8] Mollica M, Maffucci R, Lavoretano S et al. Non-small cell lung cancer presenting as "psoas muscle syndrome". Monaldi Arch Chest Dis. 2019; 89.

[9] Tsuchiyama K, Ito H, Seki M et al. Advanced bladder cancer with malignant psoas syndrome: A case report with a focus on physical findings and complications. Urol Case Rep. 2019; 26: 100958.

[10] Yamaguchi T, Katayama K, Matsumoto M. Successful Control of Pain from Malignant Psoas Syndrome by Spinal Opioid with Local Anesthetic Agents. Pain Pract. 2018; 18: 641-646.

[11] Kharbach Y, Alaoui SR, Khallouk A. Ovarian adenocarcinoma metastasis mimicking psoas abscess on imaging: a case report. Pan Afr Med J. 2020 Jul 29; 36: 231.

[12] Pearson CM. Incidence and type of pathologic alterations observed in muscle in a routine autopsy survey. Neurology. 1959; 9: 757-766.

[13] Razak AR, Chhabra R and Hughes A. Muscular metastasis, a rare presentation of non-small-cell lung cancer. Med Gen Med. 2007; 9: 20-25.

[14] The psoas muscle. Wikipedia.

[15] Jaeckle K. Nerve plexus lesions. Neurol Clin. 1991; 9: 857-866.

[16] Lievens Y, Guckenberger, M, Gomez D et al. Defining oligometastatic disease from a radiation oncology perspective: An ESTRO-ASTRO consensus document. Radiother Oncol. 2020; 148: 157-66.

[17] Agar M, Broadbent A, Chye R. The management of malignant psoas syndrome: case reports and literature review. J Pain Symptom Manage. 2004; 28: 282-293. 